\title{
Leitura compreensiva e utilização de estratégias de aprendizagem em alunos de Psicologia
}

\author{
Reading comprehension and learning strategies \\ in Psychology students
}

\author{
Acácia Aparecida Angeli dos SANTOS \\ Claudette Maria Medeiros VENDRAMINI' \\ Adriana Cristina Boulhoça SUEHIRO2 \\ Ligia Angeli Dias dos SANTOS ${ }^{3}$
}

\begin{abstract}
Resumo
Este estudo objetivou comparar o nível de compreensão em leitura percebido por alunos de Psicologia, as estratégias de aprendizagem utilizadas na superação dos problemas encontrados, bem como verificar a real dificuldade de compreensão dos alunos para responder ao teste de Cloze. Participaram 178 universitários, sendo 113 do $1^{\circ}$ ano e 65 do $4^{\circ}$ ano. $O$ procedimento foi aplicado numa única sessão e consistiu de (a) leitura de um texto científico da área, (b) questões sobre as dificuldades percebidas e sobre as estratégias utilizadas para a sua superação e (c) aplicação de um teste de cloze em texto similar ao primeiro, visando à avaliação da compreensão em leitura. Observou-se diferença estatisticamente significativa no nível de compreensão em leitura com superioridade dos alunos do $4^{\circ}$ ano em relação aos do $1^{\circ}$ ano. Contudo os alunos do $4^{\circ}$ ano perceberam-se com maior dificuldade na compreensão do primeiro texto, embora a diferença entre as turmas não tenha sido significativa. Os tipos de dificuldades de compreensão apontadas e as estratégias utilizadas pelos alunos apresentaram diferenças qualitativas entre as turmas.
\end{abstract}

Palavras-chave: compreensão de leitura; desempenho acadêmico; teste de Cloze.

\begin{abstract}
This study has aimed the comparison of reading comprehension levels and learning strategies used for overcoming eventual problems among Psychology students; as well as it verified the students'real comprehension difficulties to answer the cloze test. 178 university students (113 from the $1^{\text {st }}$ year and 65 from the $4^{\text {th }}$ year) participated in this study. The procedure was applied in one single session and consisted by: (a) reading of the area scientific text; (b) questions about the realized difficulties and the used strategies to overcome the problem; and (c) the Cloze testing application, using a text similar to the first one, in order to evaluate reading comprehension. A significant statistical difference has been observed about the reading comprehension level, as the $4^{\text {th }}$ year students have presented a better performance, compared to the students from the $1^{\text {st }}$ year. The $4^{\text {th }}$ year students acknowledged a greater difficulty in understanding the first text, although the difference between the two groups was not significant. The difficulty kinds and the strategies used by the students pointed out, qualitative differences between these groups.
\end{abstract}

Key words: reading comprehension; academic achievement; Cloze testing.

1 Professoras Doutoras, Curso de Psicologia e Programa de Pós-Graduação Stricto Sensu em Psicologia, Universidade São Francisco. R. Alexandre Rodrigues Barbosa, 45, 13251-040, Itatiba, SP, Brasil. Correspondência para/Correspondence to: A.A.A.SANTOS. E-mail: <acacia.santos@saofrancisco.edu.br>, <acaciasantos@terra.com.br>.

2 Doutoranda, Programa de Pós-Graduação Stricto Sensu em Psicologia, Universidade São Francisco. Itatiba, SP, Brasil. Bolsista Capes, SP, Brasil.

3 Psicóloga, Consultora de Recursos Humanos. 
A leitura constitui importante ferramenta de aprendizagem e ocupa papel de grande valor na vida do indivíduo, uma vez que favorece sua inserção cultural e social. Especialmente para o universitário o domínio da leitura é muito relevante, visto ser por meio dela que se dá o acesso ao conteúdo de diversas disciplinas. No entanto, a dificuldade de compreensão em leitura tem sido um obstáculo para alunos do ensino superior, pois limita a aprendizagem por meio de textos, que são as principais fontes de informação dos estudantes (Applebee, Langer \& Mullis, 1989; Armbruster \& colaboradores, 1990; Straw \& Sadowy, 1990; Smith, 1997; Santos \& Oliveira, 2004).

No Brasil, os cursos de graduação permitem o ingresso direto à profissionalização e são a última oportunidade formal de tornar o cidadão um leitor competente. Tal competência engloba a compreensão dos textos lidos criticamente, de forma que as informações obtidas possam ser utilizadas para compor uma prática profissional eficiente (Witter, 1997).

Os modelos recentes explicativos do processo de compreensão em leitura sustentam que o leitor utiliza várias fontes de conhecimento para construir o significado de um texto, incluindo sua cultura e experiências (Spiro, 1980; Robeck \& Wallace, 1990; Kintsch, 1994; Spivey, 1997). No mesmo sentido, Spires e Donley (1998) afirmam que pesquisas têm demonstrado que leitores que possuem níveis altos de conhecimento exibem uma melhor compreensão e retenção de textos que os leitores com baixo nível de conhecimento.

Todavia, de acordo com Gurthrie, Meter, Hancock, Alao, Anderson e McCann (1998), a diferença entre bons e maus leitores parece não se resumir apenas à quantia disponível de conhecimento, mas também à forma como tais conhecimentos são utilizados para facilitar a compreensão de um texto. Quando os estudantes trazem seus conhecimentos pessoais para o contexto escolar, interessam-se mais pelos textos acadêmicos e passam a construir e a manter uma relação mais significativa com a tarefa de leitura. Essa relação se constrói com o uso motivado de conhecimentos, que se expandem com o passar do tempo e ampliam as chances do estudante realizar
Referindo-se à leitura, Chance (1985) aponta para a importância de uma avaliação diagnósticoprescritiva do aluno, que permita que o seu nível de compreensão seja identificado e que procedimentos educacionais mais adequados sejam empregados. Estudos brasileiros têm mostrado que mesmo no ensino superior é possível implementar programas de intervenção que permitam a superação das deficiências em compreensão de leitura trazidas pelos alunos das etapas anteriores de sua escolarização. Ressaltam também o valor da formação de atitudes positivas diante da leitura, o que por si só pode propiciar o favorecimento de melhores condições de estudo e conseqüente desempenho acadêmico (Santos, 1997; Pellegrini, Santos \& Sisto, 2002; Sampaio \& Santos, 2002).

Nesse sentido, Bormuth (1968) considera para a avaliação da compreensão a porcentagem de acertos obtida quando se responde a um teste de Cloze, classificando-a em três níveis, a saber: (a) frustração, com menos de $44 \%$ de respostas corretas; (b) instrucional, entre 44\% e 56\% de respostas corretas e (c) independente, com acertos superiores a $56 \%$. Estudos que adotaram esse critério têm demonstrado que muitos universitários encontram-se no nível inferior de compreensão em leitura, o que poderia explicar as dificuldades acadêmicas que apresentam (Alvarez, 1990; Pellegrini, 1996; Silva \& Santos, 2004).

Diante dessa conjuntura, verifica-se a necessidade de utilização de formas eficazes de diagnóstico do nível da compreensão em leitura no início do curso superior. Uma das técnicas que tem sido considerada valiosa para esse tipo de diagnóstico é o teste de Cloze, tendo em vista que, além de ser de fácil elaboração, pode ser aplicável em textos com assuntos específicos de diferentes disciplinas (Taylor, 1953; Condemarín \& Milicic, 1988; Santos, Primi, Vendramini \&Taxa, 2002; Oliveira, 2003; Oliveira, Santos \& Primi, 2003; Santos, Suehiro, \& Oliveira, 2004).

No entanto, é importante ressaltar que o mero diagnóstico das dificuldades apresentadas pelos estudantes não é suficiente para garantir a sua superação. Ao lado disso, é indispensável identificar as estratégias utilizadas ao estudar, de modo a aumentar as possibilidades de sucesso. Ao ser detectado que o universitário não possui o repertório 
de estratégias necessárias para a apropriação dos novos conteúdos com os quais se depara, é importante que medidas preventivas e remediativas sejam tomadas. A utilização adequada de estratégias de aprendizagem vem sendo apontada por alguns estudiosos como uma forma importante de ajuda aos alunos no que diz respeito ao controle e à reflexão sobre seu próprio processo de aprender (Pressley \& Levin, 1983; Brown, 1997, Santos, 1997; Santos \& Santos, 2002).

Na perspectiva da Psicologia Cognitiva, baseada na teoria do processamento humano da informação, a aquisição do conhecimento está relacionada ao desenvolvimento da capacidade de pensar sobre os próprios pensamentos, o que permite ir muito além da apreensão de noções baseada unicamente em fatos. Essa capacidade recebe o nome de metacognição e refere-se ao conhecimento do processo de conhecer, ao planejamento, predição e monitoramento do próprio processo de aprender (Wood, Mot \& Willoughby, 1998; Cardoso, 2002; Joly \& Paula, 2005). De acordo com Boruchovitch e Costa (2000), a capacidade de metacognição existe, ainda que em menor proporção, mesmo em crianças mais novas. Ressaltam também que a percepção da nãocompreensão do conteúdo lido ou explicado pelo professor tende a aumentar com o avançar da escolaridade.

Pozo (1996) complementa que a simples execução mecânica de certas habilidades ou destrezas não constitui manifestação da aplicação de estratégia de aprendizagem. Ao contrário, para que ela se produza, é necessário um planejamento dessas habilidades em uma seqüência dirigida a um fim, que só é possível mediante o desenvolvimento do metaconhecimento (conhecimento sobre seus próprios processos psicológicos), que faz com que essas habilidades sejam utilizadas de modo estratégico. Todavia, os teóricos dessa área advertem que conhecer as estratégias não é suficiente. Faz-se necessário que os estudantes compreendam como e quando utilizá-las, bem como que estejam cônscios de sua importância.

Boruchovitch (1999) mostra que investigações atuais, tais como as de Zimmerman (1986), Zimmerman e Martinez-Pons (1986) e Brown (1997), deixam evidente a importância da identificação das estratégias de aprendizagem utilizadas pelos estudantes, que podem surgir tanto espontaneamente, como em decorrência de treinamentos metódicos. O estudo de Santos, Boruchovitch, Primi, Zenorini e Bueno (2004a) demonstra a viabilidade de desenvolvimento de uma 'Escala de avaliação de estratégias de aprendizagem para universitários' (EAPU), com evidências de validade e de precisão. Assim, vale ressaltar que essa identificação permite o conhecimento dos processos cognitivos empregados pelos aprendizes bem-sucedidos e possibilita a análise dos fatores que impedem o engajamento dos alunos no uso de estratégias adequadas.

Todo esse processo complexo envolve, segundo Leffa (1996), muitos subprocessos, que vão desde o nível básico, tal como o processamento gráfico, até níveis mais refinados exigidos em algumas tarefas, como o monitoramento da própria atenção. De acordo com Santos (1997), essa complexidade é experimentada de forma mais intensa pelos universitários que ingressam na universidade com sérios déficits nas habilidades básicas de leitura e escrita. As dificuldades enfrentadas por eles criam a consciência da necessidade de ler melhor em função da grande quantidade de informação a ser assimilada em um tempo mínimo. Daí a necessidade de se lançar mão de um conjunto de estratégias que os possa ajudar a abordar as tarefas acadêmicas com mais eficácia e eficiência.

A pesquisa realizada por Wood, Motz e Willoughby (1998), com estudantes secundários e universitários, revela que os estudantes não têm experiências com essas estratégias até a escola secundária e, muitas vezes, até depois dela. Essa deficiência pode limitar a prática e a experiência que a consciência metacognitiva Ihes proporcionaria para que se tornassem usuários prósperos e flexíveis das estratégias de aprendizagem. Com base em resultados similares, o papel da metacognição no processo de compreensão da leitura é enfatizado por estudiosos como Baker e Brown (1984), Brown, Armbruster e Baker (1986) e Baker (1989), que demonstram que os alunos que monitoram sua compreensão são mais capazes de utilizar estratégias apropriadas para melhorá-la. Outros autores consideram, ainda, que leitores 
proficientes têm maior probabilidade de usar estratégias diversificadas, dentre as quais resumir, conectar informações contidas entre parágrafos, avaliar as informações obtidas e formular perguntas e hipóteses a respeito de tais informações (Fry, Weber \& DiPierro, 1978; Perfetti, 1985).

Nessa linha, a pesquisa realizada com universitários por Morles, Manola, Donís e Urquhart (1997) demonstra que há diferença significativa na utilização de estratégias diante das dificuldades de um texto. Os leitores com baixo rendimento em leitura, em oposição aos com alto rendimento, apresentam com maior freqüência dificuldades relacionadas à integração da informação e ao desconhecimento do vocabulário e não sabem resolver os problemas que encontram para compreenderem o que lêem. De acordo com os autores, os leitores com baixo rendimento em leitura parecem estar menos conscientes de suas falhas de vocabulário e de suas dificuldades, além de preferirem utilizar primordialmente a estratégia de releitura do texto para resolverem seus problemas de compreensão.

Collins e Smith (1980) consideram que os problemas de compreensão do leitor podem estar relacionados tanto ao desconhecimento de palavras, quanto à compreensão de orações, de relações entre orações e do conteúdo global do texto. No mesmo sentido, Maria e MacGinitie (1982) atribuem tais problemas às limitações de vocabulário do leitor e à sua pouca capacidade de organizar o conteúdo do texto.

A pesquisa realizada por Santos e Santos (2002) utilizou um delineamento quase-experimental para avaliar os efeitos específicos do ensino de estratégias para a aprendizagem do espanhol básico com 88 estudantes brasileiros do curso de turismo. Os resultados revelaram que no pós-teste o grau e o tipo de dificuldade encontrados pelos alunos foram significativamente diferentes. Após o programa houve um aumento na utilização de estratégias e ganhos expressivos na compreensão em leitura de textos daquela língua estrangeira.

Contudo, a realização de outros estudos que explorem mais as formas de relação que os leitores estabelecem com diferentes tipos de texto poderá ser útil para explicitar com maior clareza como o emprego de estratégias pode funcionar como elemento facilitador da compreensão da leitura. Considerando-se a importância da leitura de textos científicos que propiciem o contato com o conhecimento recentemente produzido, o presente estudo teve por objetivos: (a) comparar o nível de compreensão percebido em leitura de textos científicos, por alunos de $1^{\circ}$ e $4^{\circ}$ ano do curso de Psicologia, (b) identificar e comparar os tipos de dificuldades de compreensão existentes para a leitura desse tipo de texto, bem como as estratégias utilizadas para superá-las e (c) verificar a eficácia do teste de Cloze como instrumento de avaliação da compreensão em leitura por universitários.

\section{Método}

\section{Sujeitos}

Participaram deste estudo 178 alunos de Psicologia de uma universidade localizada numa grande cidade do interior paulista. Os estudantes eram do turno diurno, ambos os sexos, sendo que 113 eram do $1^{\circ}$ ano e tinham média de idade de 20 anos, e 65 do $4^{\circ}$ ano, com média de idade de 26 anos.

\section{Material}

Foram utilizados neste estudo: (a) um texto científico da área, com 274 palavras, intitulado "O encaminhamento de crianças para a classe especial: Possibilidades de histórias ao contrário" (Padilha \& Góes, 1995); (b) um questionário com três questões objetivas, sendo duas sobre as dificuldades encontradas na leitura de um texto científico da área (grau de dificuldade percebido e problemas que interferiram na compreensão) e uma referente às estratégias utilizadas para sua superação e (c) um outro texto, de 294 palavras, extraído de um artigo do mesmo periódico científico "O Psicólogo escolar de hoje... O fracasso escolar de sempre..." (Correa \& Collares, 1995), de tamanho e dificuldade equivalentes ao primeiro, que foi preparado segundo os padrões tradicionais do teste de Cloze. Tal como aqui utilizado, o Cloze refere-se à estruturação de um texto, do qual se omite todo $5^{\circ}$ 
vocábulo, que é substituído por um traço de tamanho equivalente ao da palavra omitida. 0 respondente deverá completar as lacunas com a palavra que julgar mais adequada para completar o sentido do texto.

A correção foi realizada com base na proposta de correção literal (Bitar, 1989), que considera corretas somente as respostas que contêm palavras idênticas às omitidas. A pontuação máxima que poderia ser obtida era de 26 pontos, correspondente ao número de lacunas contidas no texto.

\section{Procedimento}

A aplicação foi realizada de forma coletiva em cada uma das turmas, em um único dia. Depois de explicados os objetivos do estudo, foi entregue o primeiro texto aos alunos que concordaram em participar da pesquisa. Após a leitura silenciosa do texto e, tendo-o por base, os participantes responderam às questões do questionário sobre os tipos de dificuldades encontradas e sobre as estratégias utilizadas para lidar com elas. Na seqüência, preencheram o teste de Cloze do segundo texto.

\section{Resultados}

Os resultados foram analisados em função dos objetivos e utilizaram-se estatísticas descritivas e inferenciais para o tratamento dos dados obtidos. A Figura 1 apresenta a porcentagem de universitários de $1^{\circ}$ e $4^{\circ}$ ano do curso de Psicologia, considerando a proporção de acertos obtidos no teste de Cloze.

A proporção de acertos obtida foi abaixo da esperada, considerando-se os níveis de compreensão definidos por Bormuth (1968) tanto para os alunos iniciantes (média de acertos $=46 \%$ ) quanto para os alunos do final de curso (média de acertos $=53 \%$ ). Ambos são classificados como incluídos no nível instrucional, porém estando os de $1^{\circ}$ ano no limite inferior da faixa e os do $4^{\circ}$ ano no nível superior. No entanto, considerando-se o valor da pontuação bruta, pelo teste $t$ de Student verificou-se diferença estatisticamente significativa entre as duas turmas $(t=-5,11 ; p<0,0001)$ nos escores obtidos no teste de Cloze.
Na Figura 2 apresenta-se a porcentagem de universitários em razão do grau de dificuldade percebido. Vale lembrar que os alunos poderiam indicar as alternativas nenhuma, pouca, média ou muita dificuldade na compreensão do texto científico lido.

Como pode ser observado, os alunos do $4^{\circ}$ ano declararam ter percebido maior grau de dificuldade na compreensão do primeiro texto do que os alunos iniciantes. No entanto, a diferença entre as turmas não foi estatisticamente significativa $\left(\chi^{2}[3, N=178]=5,91\right.$; $p=0,1159$ ).

Comparando-se a distribuição de freqüências dos alunos de $1^{\circ}$ e $4^{\circ}$ ano quanto às categorias de dificuldades apontadas, observa-se a existência de diferenças significativas em ambos os grupos, constatadas pelo teste $Q$ de Cochran. Para os do $1^{\circ}$ ano obteve-se $(Q[5, N=113]=70 ; p<0,0001)$ e para os do $4^{\circ}$ ano $(Q[5, N=65]=27,99 ; p<0,0001)$.

NaTabela 1 são apresentadas as porcentagens das respostas de ambos os grupos e as diferenças em cada categoria, submetidas ao teste Qui-quadrado.

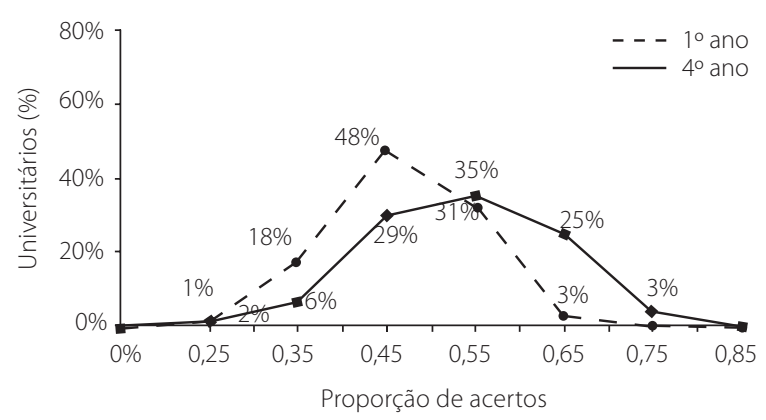

Figura 1. Nível de compreensão em leitura de textos científicos segundo proporção de acertos no teste de Cloze.

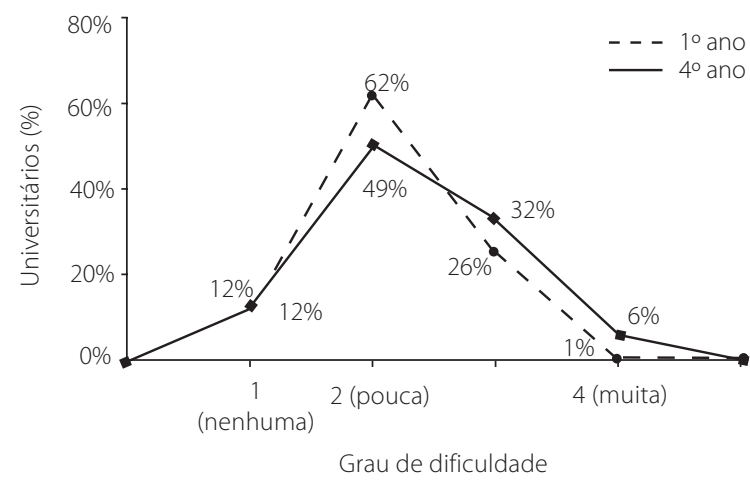

Figura 2. Grau de dificuldade declarada pelos universitários para a compreensão do texto. 
Pelos resultados obtidos, observa-se uma diferença significativa entre os alunos de $1^{\circ}$ e $4^{\circ}$ ano no que se refere às categorias "palavras sem sentido no texto" $\left(\chi^{2}[1, N=16]=11,23 ; p=0,0008\right)$ e "falta de conexão entre orações" ( $c^{2}[1, N=35]=13,04$; $p=0,0003$ ). As outras alternativas não apresentaram diferença entre as turmas.

A distribuição de freqüência de utilização das estratégias para superação das dificuldades encontradas para a compreensão do texto foi significativamente diferente. Assim, para os alunos do 10 ano obteve-se (Q[7, $\mathrm{N}=113]=164,13 ; p<0,0001)$ e para os do $4^{\circ}$ ano $(\mathrm{Q}[7, \mathrm{~N}=65]=141,20 ; p<0,0001)$, (Tabela 2).

De acordo com os resultados, a estratégia utilizada com maior freqüência tanto pelos concluintes $(81,5 \%)$ como pelos ingressantes
$(72,6 \%)$ foi a de "reler a oração completa". No entanto, a única estratégia cuja diferença de utilização foi estatisticamente significativa entre ingressantes e concluintes foi a de "relacionar a palavra ou oração com conhecimentos prévios (não presentes no texto) $\left.\chi^{2}[1, \mathrm{~N}=71]=10,26 ; p=0,0014\right)$.

\section{Discussão e Considerações Finais}

Na universidade a leitura é considerada habilidade essencial para o processo ensinoaprendizagem, posto que é, principalmente, por meio dela que se dá o acesso ao conteúdo das diversas matérias. Ao lado disso, destacam-se como muito importantes a compreensão de textos científicos e o conhecimento sobre as dificuldades inerentes a esse tipo de leitura.

Tabela 1. Comparação entre o número de respostas dadas pelos ingressantes e concluintes quanto às dificuldades apontadas para a compreensão do texto.

\begin{tabular}{|c|c|c|c|c|c|c|c|}
\hline \multirow{2}{*}{ Dificuldades apontadas } & \multicolumn{2}{|c|}{ Ingressantes } & \multicolumn{2}{|c|}{ Concluintes } & \multirow{2}{*}{$\chi^{2}$} & \multirow{2}{*}{$\begin{array}{l}\text { Graus de } \\
\text { liberdade }\end{array}$} & \multirow{2}{*}{$\begin{array}{c}\text { Significância } \\
(p)\end{array}$} \\
\hline & $\mathrm{n}$ & $\%$ & $n$ & $\%$ & & & \\
\hline Palavras desconhecidas & 13 & 11,5 & 3 & 4,6 & 2,39 & 1 & 0,1218 \\
\hline Palavras sem sentido no texto & 4 & 3,5 & 12 & 18,5 & 11,23 & 1 & 0,0008 \\
\hline Orações que não conseguiu interpretar & 48 & 42,5 & 24 & 36,9 & 0,53 & 1 & 0,4672 \\
\hline $\begin{array}{l}\text { Incoerência entre a interpretação de } \\
\text { duas ou mais orações }\end{array}$ & 18 & 15,9 & 12 & 18,5 & 0,19 & 1 & 0,6639 \\
\hline Falta de conexão entre orações & 13 & 11,5 & 22 & 33,8 & 13,04 & 1 & 0,0003 \\
\hline Outro tipo de problema & 13 & 11,5 & 10 & 15,4 & 0,55 & 1 & 0,4574 \\
\hline Total de alunos & 113 & - & 65 & - & - & - & - \\
\hline
\end{tabular}

Tabela 2. Estratégias utilizadas pelos ingressantes e concluintes para lidar com as dificuldades encontradas no texto lido.

\begin{tabular}{|c|c|c|c|c|c|c|c|}
\hline \multirow{2}{*}{ Estratégias utilizadas } & \multicolumn{2}{|c|}{ Ingressantes } & \multicolumn{2}{|c|}{ Concluintes } & \multirow[t]{2}{*}{$\chi^{2}$} & \multirow{2}{*}{$\begin{array}{l}\text { Graus de } \\
\text { liberdade }\end{array}$} & \multirow{2}{*}{$\begin{array}{c}\text { Significância } \\
(p)\end{array}$} \\
\hline & $n$ & $\%$ & $n$ & $\%$ & & & \\
\hline Reler a oração completa & 82 & 72,6 & 53 & 81,5 & 1,81 & 1 & 0,1782 \\
\hline Reler várias orações & 30 & 26,5 & 20 & 30,8 & 0,36 & 1 & 0,5464 \\
\hline $\begin{array}{l}\text { Relacionar a palavra ou oração com conhecimentos } \\
\text { prévios (não presentes no texto) }\end{array}$ & 35 & 31,0 & 36 & 55,4 & 10,26 & 1 & 0,0014 \\
\hline $\begin{array}{l}\text { Relacionar a palavra ou oração com conceitos mais } \\
\text { abrangentes (presentes no texto) }\end{array}$ & 33 & 29,2 & 18 & 27,7 & 0,05 & 1 & 0,8300 \\
\hline $\begin{array}{l}\text { Buscar o significado da palavra a partir de sua } \\
\text { estrutura }\end{array}$ & 24 & 21,2 & 11 & 16,9 & 0,49 & 1 & 0,4855 \\
\hline Formular uma hipótese para o significado da palavra & 22 & 19,5 & 7 & 10,8 & 2,29 & 1 & 0,1302 \\
\hline Nenhuma estratégia & 5 & 4,4 & 1 & 1,5 & 1,06 & 1 & 0,3043 \\
\hline Outra estratégia & 9 & 8,0 & 11 & 16,9 & 3,32 & 1 & 0,0684 \\
\hline Total de alunos & 113 & - & 65 & - & & & \\
\hline
\end{tabular}


Os resultados obtidos corroboraram as investigações que apontam o teste de Cloze como adequado para a identificação da compreensão em leitura de universitários. Assim, permitem afirmar que o nível de compreensão está aquém do desejável tanto para os que estão ingressando no ensino superior como para os que o estão concluindo. Tais achados foram similares aos de outros estudos que apontam os universitários como leitores imaturos, considerando a etapa de escolarização em que se encontram (Santos, 1997; Santos et al. 2002; Oliveira, 2003; Santos, Suehiro, \& Oliveira, 2004; Silva \& Santos, 2004).

Sabe-se que as dificuldades de leitura compreensiva apresentadas pelos universitários são obstáculos consideráveis para sua formação profissional. No entanto, verificou-se também que é possível realizar uma avaliação diagnóstica que forneça elementos importantes para a identificação de dificuldades de leitura, tal como demonstrado em outros estudos (Chance, 1985; Witter, 1997; Sampaio \& Santos, 2002; Santos \& Oliveira, 2004).

Simultaneamente, investigou-se o quanto os estudantes percebiam sobre as próprias dificuldades na leitura de um texto científico da área e sobre as estratégias utilizadas para a sua superação, visto que essas estratégias são consideradas indispensáveis para que ocorra a leitura compreensiva. Dessa forma, os dados deste estudo sustentam o que tem sido observado por outros autores sobre a pouca familiaridade dos alunos com o uso de estratégias de aprendizagem, uma vez que seria esperado que os estudantes lançassem mão de uma ampla gama de estratégias para superarem os problemas encontrados (Boruchovitch, 1999; Joly \& Paula, 2005; Pozo, 1996; Wood, Motz \& Willoughby, 1998).

No que se refere ao grau de dificuldade percebido pelos alunos, o fato de os do final de curso terem declarado maior grau de dificuldade na compreensão do que os alunos iniciantes encontra suporte nas considerações de Boruchovitch e Costa (2000). As autoras consideram que a metacognição aumenta com o avançar da escolaridade, ou seja, os alunos do final de curso têm maior consciência de suas limitações do que os ingressantes. Outros estudiosos complementam e alegam que a explicação para tal fato é que alunos de etapas mais avançadas apresentam maior conhecimento dos próprios processos de conhecer, sendo mais capazes de executar as tarefas de prever, planejar e monitorar o próprio processo de aprender (Wood, Nist \& Willoughby, 1998; Cardoso, 2002; Joly \& Paula, 2005).

Nesse sentido, verificou-se que os alunos do final do curso utilizavam mais as estratégias metacognitivas do que os alunos ingressantes, como, por exemplo, recorrer significativamente mais à estratégia de relacionar a palavra ou oração a conhecimentos prévios não presentes no texto. Os achados deste estudo são similares aos verificados na pesquisa com universitários venezuelanos realizada por Morles, Manola, Donís e Urquhart (1997) e também corroboram a importância atribuída à metacognição por Brown (1984), Brown, Armbuster e Baker (1986), Baker (1989) e pelo estudo recente de Santos et al. (2004).

As diferenças detectadas podem ser atribuídas ao pouco conhecimento técnico apresentado pelos ingressantes. Tal suposição é coerente com os resultados do estudo de Spires e Donley (1998), demonstrando que leitores possuidores de níveis altos de conhecimento exibem, constantemente, uma melhor compreensão e retenção de textos que leitores com baixo nível de conhecimento. No entanto, Guthrie et al. (1998) vão além, afirmando que tal diferença não se deve somente à quantia disponível de conhecimento, mas à forma como os leitores utilizam esse conhecimento para facilitar a compreensão de textos. Vale lembrar aqui outros trabalhos que demonstram que os alunos chegam à universidade sem terem aprendido a utilizar as estratégias de aprendizagem necessárias a um bom desempenho acadêmico (Wood, Nist \& Willoughby, 1998; Santos \& Santos, 2002).

Embora os objetivos do estudo tenham sido atingidos, é importante considerar suas limitações metodológicas, especialmente no que se refere ao tamanho da amostra e à sua circunscrição a um único curso de uma única instituição. No entanto, vale lembrar que a instituição na qual o estudo foi desenvolvido recebe alunos de várias regiões do estado de São Paulo e possui um dos primeiros cursos de Psicologia instalados no Brasil. 
Mesmo assim, entende-se como necessário que outros trabalhos sejam realizados, focalizando estudantes de outros cursos, de diferentes áreas e instituições. Apesar dos limites da pesquisa, pode-se afirmar que existem diferenças entre os alunos que estão começando e que estão concluindo o curso de Psicologia. Entretanto, na presente pesquisa observou-se que os estudantes não se diferenciaram quer no que se refere às dificuldades percebidas, quer no uso de estratégias de aprendizagem. Seria esperado que tal diferença fosse acentuada visto que ao longo do curso os alunos deveriam ter incorporado novas estratégias de aprendizagem. Embora os estudantes iniciantes e concluintes não tenham mudado de nível de compreensão, se for tomada como parâmetro a classificação de Bormuth (1968), a habilidade de compreensão em leitura aumentou significativamente, sendo considerada a comparação entre o escore total obtido por cada turma no teste de Cloze.

Finalmente, vale destacar que ler compreensivamente, ter consciência das próprias dificuldades e utilizar estratégias adequadas para superá-las são habilidades desejáveis e necessárias tanto na vida acadêmica como na profissional independentemente da área de atuação. A cada dia torna-se mais urgente que as instituições superiores se conscientizem das limitações típicas dos seus ingressantes. Assim, poderiam considerar, com mais atenção, a necessidade de programas que possibilitassem aos alunos suplantarem suas dificuldades. Essa é uma alternativa que começa a ser implementada em algumas instituições que já proporcionam, como atividade curricular ou extracurricular, espaços regulares de remediação para os estudantes que nelas ingressam sem possuírem as habilidades necessárias para a realização de um curso superior. Dessa forma, estão, de fato, contribuindo para a formação de seus alunos, tornando-os participantes ativos da sociedade com as competências específicas da profissão que escolheram.

\section{Referências}

Applebee, A. N., Langer, J. A., \& Mullis, I. V. S. (1989). Crossroads in American education. Princeton: 90 Educational Testing Service.
Alvarez, M. S. (1990). Entrenamiento en comprensión lectora utilizando la tecnica de Cloze con estudiantes del primer semestre de educación superior. Transinformação, 2, 99-113.

Armbruster, B., Anderson, T., Armstrong, J., Wise, M., Janisch, C., \&. Meyer, L. (1990). Reading and questioning in content area lessons (Technical Report n.502). Champaign: University of Illinois at Urbana - Champaign, Center for the Study of Reading.

Baker, L. (1989). Metacognition, comprehension monitoring and the adult reader. Educational Psychology Review, 1, 3-38.

Baker, L., \& Brown, A. L. (1984). Metacognitive skills and reading. In P. D. Pearson, R. Barr, M. Kamil \& P. Monsenthal (Orgs.). Handbook of reading research (pp.353-394). New York: Longman.

Bitar, M. L. (1989). Eficiência dos instrumentos de avaliação em leitura. Dissertação de mestrado não-publicada, Pontifícia Universidade Católica de São Paulo.

Bormuth, J. R. (1968). Cloze test readability: criterion references scores. Journal of Educational Measurement, 5, 189-196.

Boruchovitch, E. (1999). Estratégias de aprendizagem e desempenho escolar: Considerações para a prática educacional. Psicologia: Reflexão e Crítica, 12 (2), 361-376.

Boruchovitch, E., \& Costa, E. R. (2000). Fatores que influenciam o uso de estratégias de aprendizagem. Psico-USF, 5 (1), $11-24$.

Brown, A. L. (1997). Transforming school into communities of thinking and learning about serious matters. American Psychologist, 52 (4), 399-413.

Brown, A. L., Armbruster, B. B., \& Baker, L. (1986). The role of metacognition in reading and studying. In J. Orasanu (Org.). Reading comprehension: from research to practice (pp.49-78). Hillsdale: Erlbaum.

Cardoso, L. R. (2002). Uso de estratégias de aprendizagem e suas relações com metas de realização: um estudo no ensino superior. Dissertação de mestrado não-publicada, Universidade Estadual de Londrina.

Chance, L. (1985). Use cloze encounters of the readability kind for secondary school students. Journal of Reading, 29,690-693.

Collins, A., \& Smith, E. (1980). Teaching the process of reading comprehension (Technical Report n.182). Urbana: Center for the Study of Reading.

Condemarín, M., \& Milicic, N. (1988). Test de Cloze: procedimiento para el desarrollo y la evaluación de la comprensión lectora. Santiago-Chile: Editorial Andres Bello.

Correa, M. A. M., \& Collares, C.A.L. (1995). O psicólogo escolar de hoje... O fracasso escolar de sempre. Trajetos, 2 (3), 79-96.

Fry, E., Weber, J., \& DiPierro, J. (1978). A partial validation of the kernel distance theory for readability. In P. D. Pearson \& J. Hansen (Orgs.). Reading: disciplined inquiry in process and practice (pp.121-124). Clemson: National Reading Conference. 
Gurthrie, J. T., Meter, P. V., Hancock, G. R., Alao, S., Anderson, E., \& McCann, A. (1998). Does concept-oriented reading instruction increase strategy: use and conceptual learning from text? Journal of Educational Psychology, 90 (2), 261-278.

Joly, M. C. R. A., \& Paula, L. M. (2005). Avaliação do uso de estratégias de aprendizagem e a compreensão em leitura de universitários. In M. C. R. A. Joly, A. A. A. Santos, \& F. F. Sisto. (Orgs.). Questões do cotidiano universitário (pp.3358). São Paulo: Casa do Psicólogo.

Kintsch, W. (1994). Text comprehension, memory and learning. American Psychologist, 49 (4), 294-303.

Leffa, V. J. (1996). Fatores da compreensão na leitura. Cadernos do IEL, 15 (15), 143-159.

Maria, K., \& MacGinitie, W. H. (1982). Reading comprehension disabilities: Knowledge, structure and nonaccommodating text processing strategies. Annals of Dyslexia, 32, 33-59.

Morles, A., Manola, A., Donís, Y., \& Urquhart, R. (1997). Resolución de problemas de procesamiento de la información durante la lectura. Lectura y Vida, 18 (3), 13-22.

Oliveira, K. L. (2003). Compreensão de leitura, desempenho acadêmico e avaliação da aprendizagem em universitários. Dissertação de mestrado não-publicada, Programa de Estudos Pós-graduados em Psicologia. Universidade São Francisco, Itatiba-SP.

Oliveira, K. L., Santos, A. A. A., \& Primi, R. (2003). Estudo das relações entre compreensão em leitura e desempenho acadêmico na universidade. Interação, 7 (1), 19-25.

Padilha, A. M., \& Góes, C. (1995). O encaminhamento de crianças para classe especial: possibilidades de histórias ao contrário. Trajetos, 2 (3), 14-23.

Pellegrini, M. C. K. (1996). Avaliação dos níveis de compreensão e atitudes frente à leitura em universitários. Dissertação de mestrado não-publicada, Programa de Estudos Pósgraduados em Educação da Universidade São Francisco. Bragança Paulista.

Pellegrini, M. C. P., Santos, A. A. A., \& Sisto, F. F. (2002). Evaluación de las actitudes de lectura en universitarios. Lectura y Vida, 23 (2), 26-33.

Perfetti, C.A. (1985). Reading ability. New York: Oxford University Press.

Pozo, J. I. (1996). Estratégias de aprendizagem. In C. Coll, J. Palácios \& A. Marchesi (Orgs.). Desenvolvimento psicológico e educação: psicologia da educação, 2 (pp.176197). Porto Alegre: Artes Médicas.

Pressley, M., \& Levin, J. R. (1983). Cognitive strategy research: Psychological foundations. New York: Springer-Verlag.

Robeck, M. C., \& Wallace, R. R. (1990). The psychology of reading: an interdisciplinary approach. New Jersey: Erlbaum.

Sampaio, I. S., \& Santos, A. A. A. (2002). Leitura e redação entre universitários: Avaliação de um programa de intervenção. Psicologia em Estudo, 7 (1), 31-38.

Santos, A. A. A., \& Santos, E. C. (2002). Avaliação de um programa de intervenção para o ensino do espanhol, Caracas-VE. Paradigma, 23 (2), 49-75.
Santos, A. A. A. (1997). Psicopedagogia no $3^{\circ}$ grau: Avaliação de um programa de remediação em leitura e estudo. ProPosições, 8 (1), 27-37.

Santos, A. A. A., \& Oliveira, K. L. (2004). A importância da compreensão em leitura para a aprendizagem de universitários. In E. Boruchovitch \& J. A. Bzuneck (Orgs.). Aprendizagem: processos psicológicos e o contexto social na escola (pp.119-148). Petrópolis: Vozes.

Santos, A. A. A., Primi, R., Vendramini, C. M. M., \&Taxa, F. (2002). OTeste de Cloze na avaliação da compreensão em leitura. Psicologia: Reflexão e Crítica, 15 (3), 401-417.

Santos, A. A. A., Boruchovitch, E., Primi, R., Zenorini, R. P. C., \& Bueno, J. M. H (2004). Escala de avaliação de estratégias de aprendizagem para universitários (EAP-U): aplicação do Modelo de Rasch de créditos parciais. Psicologia: Teoria, Investigação e Prática, 9 (2), 227-242.

Santos, A. A. A., Suehiro, A. C. B., \& Oliveira, K. L. (2004). Habilidades em compreensão da leitura: um estudo com alunos de Psicologia. Estudos de Psicologia, 21 (2), 29 -42.

Silva, M. J. M., \& Santos, A. A. A. (2004). A avaliação da compreensão em leitura e o desempenho acadêmico de universitários. Psicologia em Estudo, 8 (3), 459-467.

Smith, F. (1997). Reading without nonsense. Toronto: Teacher College Press.

Spires, H. A., \& Donley, J. (1998). Prior knowledge activation: Inducing Engagement with informational texts. Journal of Educational Psychology, 90 (2), 249-260.

Spiro, R. J. (1980). Constructive processes in prose comprehension and recall. In R. J. Spiro, B. C. Bruce \&W. F. Brewer (Orgs.). Theoretical issues in reading comprehension (pp.245-278). Hillsdale: Erlbaum.

Spivey, N. (1997). The constructivist metaphor. Boston: Academic Press.

Straw, S., \& Sadowy, P. (1990). Dynamics of communication: Transmission, translation and interaction in reading comprehension. In D. Bogdan \& S. Straw (Orgs.). Beyond communication: reading, comprehension and criticism (pp.21-47). Portmouth: Boynton \& Cook.

Taylor, W. L. (1953). Close procedure: a new tool for measuring readability. Journalism Quarterly, 30, 415-433.

Witter, G. P. (1997). Psicologia, leitura e universidade. Campinas: Alínea.

Wood, E., Mot, M., \& Willoughby, T. (1998). Examining student's retrospective memories of strategy development. Journal of Educational Psychology, 90 (4), 698-704.

Zimmerman, B. J. (1986). Becoming a self-regulated learner: which are the key subprocesses? Contemporary Educational Psychology, 1, 307-313.

Zimmerman, B. J., \& Martinez-Pons, M. (1986). Development of a structured interview for assessing student use of selfregulated learning strategies. American Educational Research Journal, 23, 614-628.

Recebido em: 26/8/2005

Aprovado - 1a forma em: 14/10/2005 